\title{
A Case of C-ANCA Associated Retroperitoneal Fibrosis
}

\author{
Vinay Srinivasa*, Alok Gupta, Toby Brunckhorst, Marcus Dickey and Brent Simkus
}

Toowoomba Hospital, Pechey St, South Toowoomba QLD, Australia

*Corresponding author: Vinay Srinivasa, Toowoomba Hospital, Pechey St, South Toowoomba QLD, Australia, E-mail: docvgs@yahoo.com.au

Received: 24 Sep, 2020 | Accepted: 17 Oct, 2020 | Published: 22 Oct, 2020

Citation: Srinivasa V, Gupta A, Brunckhorst T, Dickey M, Simkus B (2020) A Case of C-ANCA Associated Retroperitoneal Fibrosis. Int J Nephrol Kidney Fail 6(4): dx.doi.org/10.16966/2380-5498.202

Copyright: (C) 2020 Srinivasa V, et al. This is an open-access article distributed under the terms of the Creative Commons Attribution License, which permits unrestricted use, distribution, and reproduction in any medium, provided the original author and source are credited.

\section{Abstract}

Granulomatosis with Polyangitis (GPA) is a type of small vessel vasculitis that has a prevalence rate of 25-160 cases per million population, and an incidental rate of 0.4 cases per 100,000 population per year. Clinical features of the disease involve the respiratory and renal systems. However, large vessels such as the aorta and retroperitoneal tissue are rarely involved.

We present the case of a middle-aged male who presented with an obstructive nephropathy in which abdominal CT revealed a soft tissue mass encompassing the aorta and inferior vena cava causing obstruction of the left ureter. Despite ureteric stenting, serum creatinine failed to improve. Furthermore, urinalysis demonstrated an active urinary sediment; hemoproteinuria. Serum c-ANCA and PR-3 antigen titres were positive. Renal biopsy was performed and confirmed pauci-immune vasculitis.

Our patient was induced with pulsed intravenous methylprednisolone and cyclophosphamide and as part of his maintenance treatment received prednisolone and oral cyclophosphamide.

On follow up, partial remission has been achieved with his serum creatinine returning to baseline level and proteinuria reduced, though erythrocytes are still evident on urine microscopy. Repeat abdominal imaging, has revealed reduction in the size of the soft tissue mass with treatment.

Biopsy proven vasculitis has been shown in patients with retroperitoneal fibrosis. Few case reports and series have described this association, inferring a pathogenic role of ANCA in the development of retroperitoneal fibrosis. Moreover it has been suggested that retroperitoneal fibrosis may be an early clinical manifestation of ANCA associated vasculitis. Consequently, ANCA associated vasculitis should be considered in the differential diagnosis of any patient who has retroperitoneal fibrosis and active urinary sediment.

Keywords: Vasculitis; ANCA; Glomerulonephritis; Retroperitoneal fibrosis

\section{Introduction}

Vasculitis encompasses a broad group of syndromes characterised by inflammation of the blood vessels [1-3].

The classification of vasculitis is based on the Chapel Hill consensus conference classification system, which considered vessel size and distribution along with pathogenesis [4].

ANCA Associated Vasculitis (AAV) is a necrotising small-vessel vasculitis associated with diseases such as Granulomatosis with polyangiitis (GPA), Microscopic Polyangiitis (MPA) \& Eosinophilic Granulomatosis with polyangiitis (EGPA) [4].

Positive antibodies against myeloperoxidase (MPO-ANCA) or proteinase 3(PR3-ANCA) are detected in serum [5].

GPA previously known as Wegener's Disease is a rare disease with a prevalence of 25-160 cases per million populations and an incidence of 0.4 cases per 100,000 population/year [6].
It is more common in men of Caucasian origin aged over 40 years, although it can appear at any age. The principal feature of the disease is the involvement of the respiratory system, affected by the outset in over $90 \%$ of cases. The kidney is also affected. Other structures, such as the retroperitoneal tissue or large vessels, including the aorta, are involved, but changes in these structures are liable to be missed during clinical evaluation. This is important, because of the high associated risk of obstruction of local organs caused by granulomatous inflammation [6].

Here we report, a case of a middle-aged male who presented with obstructive nephropathy secondary to retroperitoneal fibrosis (RPF). Cross-sectional imaging revealed an infrarenal abdominal aortic aneurysm.

Serum showed C-ANCA positivity with elevated PR-3 antigen titers, and renal biopsy confirmed pauci-immune crescentic glomerulonephritis, ANCA associated vasculitis-related aortitis, is a rare presentation with few cases reported in the literature [5,7]. 


\section{Case History}

A 47-year-old male was referred to our facility, with a four-week history of bilateral lower limb weakness, left flank pain associated with night sweats and unintentional weight loss of twenty kilograms over four months. He reported a history of frank hemoptysis with associated sinusitis twelve months previously.

Physical examination findings on presentation included palpable inguinal lymphadenopathy and left flank tenderness on abdominal examination. No rash was present on skin examination, but neurological examination revealed distal lower limb weakness with no sensory loss consistent with a lower limb motor neuropathy.

Laboratory investigations on admission revealed a serum creatinine of $90 \mathrm{micromol} / \mathrm{L}$, and urinalysis showed active urinary sediment with $2+$ proteins on the dipstick and microscopic hematuria with greater than 190 erythrocytes.

Cross-sectional imaging of the abdomen and pelvis showed obstruction of the mid-left ureter by a soft tissue mass encompassing the aorta and inferior vena cava (Figure 1).

Furthermore, whole-body positron emission tomography scan (PET scan) demonstrated intense FDG avidity; extensive retroperitoneal soft tissue thickening; infrarenal abdominal aortic aneurysm, increased FDG avidity in the spleen and bilateral external and inguinal lymph nodes. Excisional lymph node biopsy of the inguinal nodes, bone marrow biopsy, and peripheral flow cytometry was performed to exclude a lymphoproliferative disorder. Histology and cytometry were normal. Biopsy of the aortic mass was not attempted due to the high risk of bleeding.

Treatment of hydronephrosis involved the placement of a double J stent into the left ureter to reduce the hydronephrosis. Despite this, serum creatinine worsened, peaking at $215 \mathrm{micromol} / \mathrm{L}$ (Table 1).

Further imaging; ultrasound of the kidneys, ureter, and bladder (USSKUB); showed moderate dilatation of the right pelvicalyceal system. Consequently, a double J stent was inserted into the right ureter. Despite this, serum creatinine was still high and did not fall. Microscopic haematuria was still present, and a glomerulonephritis screen panel was ordered consisting of ANCA, Complement (C3/4), ANA, dsDNA, and ENA.

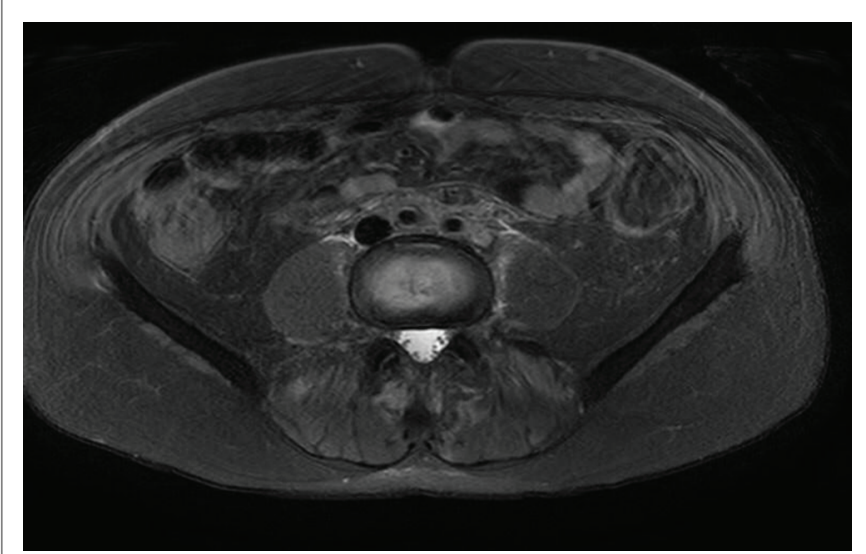

Figure 1: Cross-sectional MRI of the abdomen and pelvis, showing soft fibrous tissue thickening encompassing the aorta and inferior vena cava.
ANCA serology was positive; c-ANCA titers were elevated at 640 units, and PR3-antigen was raised, with titers greater than 1000 central units (CU) (Table 1).

The patient was pulsed with three doses of methylprednisolone and underwent a renal biopsy which confirmed pauci-immune crescentic glomerulonephritis. Twenty-two glomeruli were in the sample of which seven were cellular crescents. The tubules showed no scarring or atrophy, and immunofluorescence staining was negative.

Our patient was treated with oral prednisolone and oral cyclophosphamide as induction therapy for three months. Serum creatinine improved with a reduction in c-ANCA and PR-3 titers, and repeat urinalysis showed no hematuria or proteinuria. Repeat imaging of the genitourinary tract has shown resolution of the hydronephrosis, with the bilateral ureteric stents removed.

Repeat CT abdomen, however, still shows the periaortic thickening, though less prominent than before. Oral cyclophosphamide was ceased after three months and changed to mycophenolate with low dose prednisolone as maintenance therapy.

\section{Discussion and Conclusion}

AAV related aortitis is a rare presentation. From our literature search, only 10 cases have been reported in the literature [5].

The c-ANCA pattern is specific for granulomatosis with polyangiitis (GPA). A strong association exists between PR-3 antibodies and GPA, but tissue diagnosis is required [1-9]. Furthermore, antibodies to PR-3 are said to correlate with disease activity [8].

Biopsy proven vasculitis has been demonstrated in patients with retroperitoneal fibrosis. (RPF) [1-3,5-9]. RPF can co-exist with any kind of vasculitis including necrotising vasculitis, granulomatous vasculitis, and aortitis [8].

Case series and reports have been published describing an association between RPF and positive c-ANCA or p-ANCA vasculitis, fuelling speculation that a vasculitic process may play a role in the pathogenesis of RPF [7].

The autoimmune nature of the disease with inflammatory changes affecting small and medium-sized vessels has been proposed as a pathogenic mechanism for the development of RPF [6,7]. It has been theorised that vasculitis involves the aortic vasa vasorum and retroperitoneal vessels, thus leading to different pathological pictures, creating an aortic aneurysm with marked adventitial inflammation (the pathological hallmark of 'inflammatory aneurysms') or extending into the retroperitoneum; eliciting a fibrogenic response surrounding other structures (e.g. the ureters or duodenum) [7].

Table 1: Laboratory results of renal functions and autoantibodies during admission discharge, and on follow up.

\begin{tabular}{|l|c|c|c|c|}
\hline & Admission & $\begin{array}{c}\text { Peak } \\
\text { inpatient }\end{array}$ & Discharge & OPD Follow-up \\
\hline Urea (micromol/I) & 8.2 & 16.2 & 14 & 10 \\
\hline $\begin{array}{l}\text { Creatinine } \\
\text { (micromol/L) }\end{array}$ & 90 & 215 & 142 & 106 \\
\hline GFR (ml/min) & 82 & 17 & 23 & 65 \\
\hline c-ANCA (IU) & 640 & - & - & $<50$ \\
\hline PR3 (IU) & $>1000$ & - & - & $<40$ \\
\hline
\end{tabular}


The histological picture of RPF, inflammatory abdominal aortic aneurysms, and perianeurysmal RPF has been described to be indistinguishable, with all three considered to be within the spectrum of chronic periaortitis.

Furthermore, necrotising vasculitis of the vasa vasorum has been reported in at least one-third of inflammatory aneurysms [7]. First-line therapy should be a combination of steroids and immunosuppressive treatment, such as cyclophosphamide with a favourable response reported in the literature $[4,6,7,10]$.

Martinez-Odriozola P, et al. described a 46-year-old male with RPF causing ureteral obstruction with associated raised PR-3 ANCA titers [9].

As in our case, serum creatinine did not improve with bilateral ureteric stent insertion to relieve hydronephrosis inferring a pathogenic role of ANCA. Like Martinez-Odriozola P, et al. We also propose that a simultaneous glomerulonephritis be excluded in patients with RPF and obstructive nephropathy, who have elevated ANCA titers and active urinary sediment in whom renal functions do not improve after surgery or placement of ureteral stents [9].

Furthermore, we also suggest that RPF be included in the differential diagnosis of c-ANCA disorders. Published case reports in the literature have reported that RPF can develop as an early clinical manifestation before the appearance of classical signs of GPA [8].

Knowledge of this critical concept can thus avoid diagnostic error and enable an appropriate treatment approach to be employed at the earliest opportunity.

Our patient had clinical features that manifested early, and although there was a delay in diagnosis, a good response to immunosuppressive treatment has been achieved.

\section{Teaching Points}

- Large vessel involvement (Aorta and Vena Cava) may be associated with AAV.

- RPF may coexist with vasculitis.

- Autoimmune pathogenesis may play a role in the development of RPF.
- Treatment of RPF associated vasculitis with immunosuppressive therapy; prednisolone and cyclophosphamide have shown a favorable response.

- RPF may develop as an early clinical manifestation of GPA.

\section{References}

1. Amos LA, Roberts MA, Blair S, McMahon LP (2012) cANCAassociated aortitis. Clin Kidney J 5: 47-49.

2. Aslangul E, Ranque B, Papo T (2003) Pseudotumoral retroperitoneal fibrosis and localised vasculitis with very high serum levels of antiPR3 ANCA. Am J Med 115: 250-252.

3. Chirinos JA, Tamariz LJ, Lopes G, Carpio FD, Zhang X, et al. (2004) Large vessel involvement in ANCA- associated vasculitides: report of a case and review of the literature. Clin Rheumatol 23: 152-159.

4. Jennette JC, Falk RJ, Alba MA (2016) Nomenclature of Vasculitides: 2012 Revised International Chapel Hill Consensus Conference. Systemic Vasculitides: Current Status and Perspectives 15-28.

5. Revilla EMG, Fernandez AA, Ramirez MTR, Pardo SC, Moragues MAJ (2016) Retroperitoneal fibrosis with periaortitis: A case report of an unusual form of presentation of granulomatosis with polyangitis. Respir Med Case Rep 19: 121-124.

6. Miyawaki M, Oda S, Hirata K, Yuki H, Utsunomiya D, et al. (2017) Granulomatosis with polyangitis can cause periaortitis and pericarditis. Clin Case Rep 5: 1732-1733.

7. Skeik N, Hari G, Nasr R (2019) Aortitis caused by antineutrophil cytoplasmic antibodies (ANCA)-associated vasculitis: a case-based review. Rheumatol Int 39: 1983-1988.

8. Mavragani CP, Voulgarelis M (2007) Retroperitoneal fibrosis and c-ANCA positivity. Clin Rheumatol 26: 115-116.

9. Martinez-Odriozola P, Gutierrez- Macias A, Eguren IM, Lezama JA (2008) Association of idiopathic retroperitoneal fibrosis, rapidly progressive glomerulonephritis and antiproteinase 3 antineutrophil antibodies (anti PR3-ANCA). Clin Nephrol 70: 251-254.

10. Vaglio A, Manenti L, Allegri L, Ferrozzi F, Corradi D, et al. (2002) ANCA-positive periaortic vasculitis: does it fall within the spectrum of vasculitis? J Intern Med 251: 268-271. 